OPEN ACCESS

Edited by:

Yrsa Bergmann Sverrisdóttir University of Oxford, United Kingdom

Reviewed by:

Herbert Jelinek,

Charles Sturt University, Australia

Chloe E. Taylor,

Western Sydney University, Australia

*Correspondence:

Amirehsan Sarabadani Tafreshi sarabadani@hest.ethz.ch

Specialty section:

This article was submitted to Autonomic Neuroscience, a section of the journal

Frontiers in Physiology

Received: 24 December 2016 Accepted: 05 May 2017

Published: 02 June 2017

Citation:

Sarabadani Tafreshi A, Riener $R$ and Klamroth-Marganska V (2017) Distinctive Steady-State Heart Rate and Blood Pressure Responses to

Passive Robotic Leg Exercise during Head-Up Tilt: A Pilot Study in Neurological Patients. Front. Physiol. 8:327.

doi: 10.3389/fphys.2017.00327

\section{Distinctive Steady-State Heart Rate and Blood Pressure Responses to Passive Robotic Leg Exercise during Head-Up Tilt: A Pilot Study in Neurological Patients}

\author{
Amirehsan Sarabadani Tafreshi ${ }^{1,2 *}$, Robert Riener ${ }^{1,2}$ and Verena Klamroth-Marganska ${ }^{1,2}$ \\ ${ }^{1}$ Sensory-Motor Systems Lab, Department of Health Sciences and Technology, Institute of Robotics and Intelligent Systems, \\ ETH Zurich, Zurich, Switzerland, ${ }^{2}$ Reharobotics Group, Medical Faculty, Spinal Cord Injury Center, Balgrist University \\ Hospital, University of Zurich, Zurich, Switzerland
}

Introduction: Robot-assisted tilt table therapy was proposed for early rehabilitation and mobilization of patients after diseases such as stroke. A robot-assisted tilt table with integrated passive robotic leg exercise (PE) mechanism has the potential to prevent orthostatic hypotension usually provoked by verticalization. In a previous study with rather young healthy subjects [average age: $25.1 \pm 2.6$ years (standard deviation)], we found that PE effect on the cardiovascular system depends on the verticalization angle of the robot-assisted tilt table. In the current study, we investigated in an older population of neurological patients (a) whether they show the same PE effects as younger healthy population on the cardiovascular system at different tilt angles, (b) whether changing the PE frequency (i.e., stepping speed) influences the PE effect on the cardiovascular system, (c) whether PE could prevent orthostatic hypotension, and finally, (d) whether PE effect is consistent from day to day.

Methods: Heart rate $(\mathrm{HR})$, and systolic and diastolic blood pressures (sBP, dBP) in response to PE at two different tilt angles $\left(\alpha=20^{\circ}, 60^{\circ}\right)$ with three different PE frequencies (i.e., 0,24 , and 48 steps per minute) of 10 neurological patients [average age: $68.4 \pm 13.5$ years (standard deviation)] were measured on 2 consecutive days. Linear mixed models were used to develop statistical models and analyze the repeated measurements.

Results: The models show that: PE significantly increased $\mathrm{sBP}$ and $\mathrm{dBP}$ but had no significant effect on HR. (a) Similar to healthy subjects the effect of PE on SBP was dependent on the tilt angle with higher tilt angles resulting in a higher increase. Head-up tilting alone significantly increased $\mathrm{HR}$ and $\mathrm{dBP}$ but resulted in a non-significant drop in SBP. PE, in general, had a more additive effect on increasing BP. (b) The effect of PE was not influenced by its speed. (c) Neither during head-up tilt alone nor in combination with PE did participants experience orthostatic hypotension. (d) The measurement day was not a statistically significant factor regarding the effects of verticalization and PE on the cardiovascular response. 
Conclusion: We provide evidence that PE can increase steady-state values of sBP and $\mathrm{dBP}$ in neurological patients during head-up tilt. Similar to healthy subjects the effect on sBP depends on the verticalization angle of the robot-assisted tilt table. PE might have the potential to prevent orthostatic hypotension, but as the amount of drop in BP in response to head-up tilting was not leading to orthostatic hypotension in our patients, we could neither conclude nor reject such a preventive compensatory effect. Furthermore, we found that changing the PE speed does not influence the steady-state cardiovascular response.

Keywords: rehabilitation, robotic tilt table, orthostatic hypotension, stepping speed, exercise intensity, cardiovascular system, linear mixed models, parametric bootstrap

\section{INTRODUCTION}

Critically ill patients (e.g., after neurological diseases such as stroke or spinal cord injury, SCI) often have to stay in bed for days and weeks. This prolonged bed rest might lead to secondary complications that delay or even prevent patients' recovery (Dittmer and Teasell, 1993; Teasell and Dittmer, 1993; Brower, 2009). Mobilization can ameliorate such adverse side effects and improve functional recovery (Morris, 2007; Burtin et al., 2009; Bourdin et al., 2010). However, cardiovascular autonomic dysfunction (Ravensbergen et al., 2013; West et al., 2015) and in particular, orthostatic hypotension is prevalent among these patients (Illman et al., 2000; Feldstein and Weder, 2012; Ravensbergen et al., 2013) and is a barrier for mobilization. Head-up tilt was originally applied to investigate orthostatic hypotension as part of examining the autonomic nervous system function (Pagani et al., 1986). For such diagnostic purposes, usually mechanical head-up tilt tables were used. However, in recent years, head-up tilt tables have also been used for rehabilitation. As a potential solution for safe mobilization, a robot-assisted tilt table has been suggested (Colombo et al., 2005). Feasibility in early rehabilitation of patients with neurological disorders was demonstrated (Craven et al., 2013; Kuznetsov et al., 2013; Frazzitta et al., 2015). In contrast to conventional tilt tables, robot-assisted tilt tables (Erigo, Hocoma AG, Switzerland) have an integrated robotic module, which allows passive robotic leg exercise (PE) training (Figure 1). The PE mechanism has been utilized for developing biofeedback systems (Giggins et al., 2013) for early rehabilitation, either without contribution (Wieser et al., 2014; Sarabadani Tafreshi et al., 2015, 2017a) or with partial active participation (Laubacher et al., 2015; Saengsuwan et al., 2015a,b) of the patient in the loop, in order to drive cardiovascular variables or performance to desired levels. However, the main objective for PE in the robotassisted tilt table is the ability to enhance blood circulation and thus, avoid orthostatic hypotension (Czell et al., 2004;

\footnotetext{
Abbreviations: AIC, Akaike information criterion; BIC, Bayesian information criterion; BMI, Body mass index; BP, Blood pressure; bpm, Beats per min; CI, Confidence interval; CIP, Critical Illness Polyneuropathy; CVA, Cerebrovascular accident; dBP, Diastolic blood pressure; FES, Functional electrical stimulation; HIE, Hypoxic Ischemic Encephalopath; HR, Heart rate; LRT, Likelihood ratio test; ML, Maximum likelihood; PE, Passive robotic leg exercise; REML, Restricted maximum likelihood; sBP, Systolic blood pressure; SE, Standard error.
}

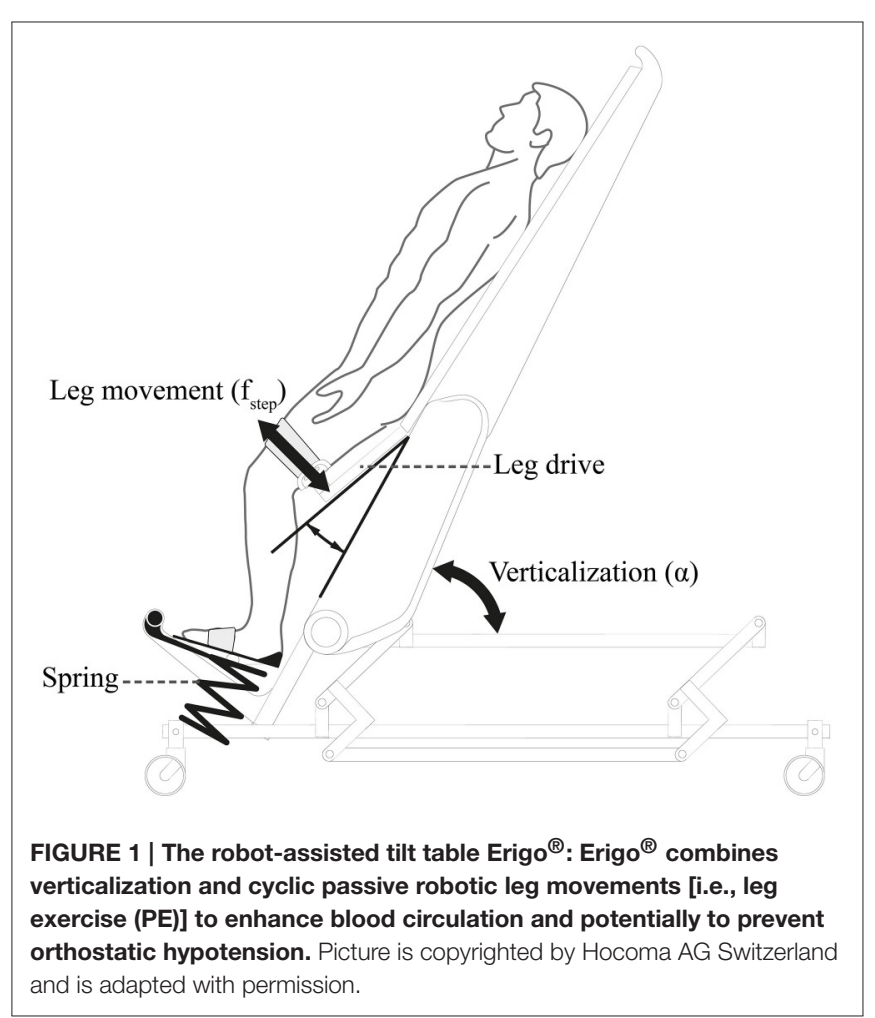

Colombo et al., 2005). Mobilization through head-up tilting alone might result in premature termination of the therapy due to orthostatic reactions leading to adverse events such as dizziness or presyncope, whereas involving $\mathrm{PE}$ might prevent such conditions and enable an effective therapy without termination (Kuznetsov et al., 2013). Moreover, training together with PE might be more efficient in improving leg strength and cerebral blood flow than head-up tilting alone without PE (Kuznetsov et al., 2013).

By definition, orthostatic hypotension is a drop of more than $20 \mathrm{mmHg}(30 \mathrm{mmHg}$ in patients with supine hypertension) in systolic blood pressure (sBP) or more than $10 \mathrm{mmHg}$ in diastolic blood pressure (dBP) (Freeman et al., 2011). The application of robot-assisted tilt tables in rehabilitation increases orthostatic tolerance and reduces the occurrence of orthostatic hypotension over time (Taveggia et al., 2015). In addition to long-term 
effectiveness, training with a robot-assisted tilt table is also effective in the short-term prevention of orthostatic hypotension and resultant syncope. Comparison of the robot-assisted tilt table with regular tilt tables has shown that it results in a lower number of syncopes in both healthy subjects and patients (Czell et al., 2004; Luther et al., 2008; Kuznetsov et al., 2013). It is known that PE evokes a cardiovascular response in healthy subjects (Chi et al., 2008) and patients (Yoshida et al., 2013). However, most of these studies have been limited in investigating the effect of PE at an arbitrarily chosen single intensity (i.e., PE frequency) and one single tilt angle in the range of $\alpha=60^{\circ}-75^{\circ}$. Although, some preliminary explorations and assumptions have been done about the effect of performing PE at different frequencies or different tilt angles (Wieser, 2011; Wieser et al., 2014), a systematic study is missing. In our previous work conducted with a rather young healthy population [average age: $25.1 \pm 2.6$ years (standard deviation)], we found that PE influences the cardiovascular system's response systematically. However, this effect is highly dependent on the angle of tilt at which PE is performed; the PE effect is not present at each angle and not always in the same direction (i.e., increase or decrease of cardiovascular variables). Thus, we concluded that the potential preventive effect of PE on orthostatic hypotension is dependent on the verticalization angle of the robot-assisted tilt table (Sarabadani Tafreshi et al., 2016). Aging (Jones et al., 2003) and neurologic diseases (Benarroch, 2008) can lead to a depressed baroreflex sensitivity. In the current work, we aimed at exploring the PE effect in an older population of neurological patients, where a depressed baroreflex sensitivity and thus, a stronger cardiovascular response to the PE or head-up tilting was expected. We hypothesized that; (a) similar to young healthy subjects, in neurological patients the $\mathrm{PE}$ effect on the cardiovascular system is tilt-angle dependent; (b) the PE frequency plays a major role in the PE effect on the cardiovascular system's response, and a higher PE frequency systematically results in a higher effectiveness; (c) the PE has a compensatory effect which can prevent orthostatic hypotension when dealing with orthostatic challenge; (d) the PE effect is systematically replicable and consistent from day to day. To examine our hypotheses, we measured heart rate (HR), sBP, and $\mathrm{dBP}$ of ten neurological patients [average age: $68.4 \pm 13.5$ years (standard deviation)] in response to PE at three different frequencies [i.e., 0, 24, and 48 steps per minute (min)] and at two different tilt angles (i.e., $\alpha=20^{\circ}, 60^{\circ}$ ), on 2 consecutive days.

It is worth mentioning that in our previous study (Sarabadani Tafreshi et al., 2016), we had also tested the effect of the simultaneous application of functional electrical stimulation (FES) to leg muscles during the PE on HR, sBP, and dBP. Since our investigation (Sarabadani Tafreshi et al., 2016) and furthermore, other patient studies investigating deployment of FES together with PE (Chi et al., 2008; Craven et al., 2013; Kuznetsov et al., 2013; Yoshida et al., 2013) had found no significant contribution from the FES to the PE effect, in the current study, we omitted investigation of the FES effect. Instead, we investigated whether changing the PE frequency (i.e., stepping speed) influences the PE effect [see Hypothesis (b) above].

\section{MATERIALS AND METHODS}

\subsection{Robot-Assisted Tilt Table}

Erigo ${ }^{\circledR}$ (Hocoma AG, Volketswil, Switzerland) is a robot-assisted tilt table designed for rehabilitation of patients facing bed rest (Colombo et al., 2005). The table is enhanced with a motordriven stepping device that can provide PE through two leg drives (Figure 1). The stepping frequency $f_{\text {step }}$ can be continuously adjusted between 0 and 80 steps per min with equal periods of extension and flexion phases. Simultaneous to the robotic leg movement, cycling leg loading is provided through two springs located beneath the patient's feet. The tilt table verticalization angle $\alpha$ can be continuously adjusted between 0 and $75^{\circ}$.

\subsection{Measurement Equipment}

The raw blood pressure (BP) signal was measured non-invasively using a CNAP ${ }^{\circledR}$ monitor 500 (CNSystems Medizintechnik AG, Austria). The monitor uses a double-finger and an arm cuff to measure the BP signal. Before each measurement, about $2 \mathrm{~min}$ for calibration are required. To record the data in real-time, the BP signal from the monitor $(100 \mathrm{~Hz})$ was fed into a g.USBamp biosignal amplifier (g.tec medical engineering $\mathrm{GmbH}$, Austria) connected to a laptop through a standard USB connection. The data was recorded using a MATLAB ${ }^{\circledR} /$ Simulink model (Mathworks Inc., Natick, MA, United States) through the provided API for the biosignal amplifier (g.tec medical engineering $\mathrm{GmbH}$, Austria).

\subsection{Subjects}

Neurological patients were recruited from Zürcher Höhenklinik Wald, a neurorehabilitation center in the metropolitan area of Zurich. The study was approved by Kantonale Ethikkommission Zürich and Swissmedic, and it was registered at ClinicalTrials.gov (registration identifier NCT02268266). Criteria for participation included post-acute phase after stroke or other neurological functional disorder, oxygen saturation of the blood of at least 92\% (measured by pulse oximetry), resting HR 40-100 beats per min (bpm), resting sBP $120-220 \mathrm{mmHg}$, no severe contraction in the legs (modified Ashworth scale $>3$ ). For more details, see clincialtrials.gov (identifier NCT02268266).

\subsection{Experimental Protocol}

Each patient was measured at two different head-up tilt angles $\left(\alpha=20^{\circ}\right.$ and $\alpha=60^{\circ}$ ) and three different PE frequencies $(0,24$, and 48 steps per $\mathrm{min})$ in a random order (Figure 2). Temporary cardiovascular changes such as overshoots could occur due to the sudden changes in the applied external stimuli (e.g., sudden change of PE frequency from 0 to 24 steps per min) (Sarabadani Tafreshi et al., 2017b). To avoid considering transient reactions in our evaluations, we focused on the steady-state responses. Transient responses to head-up tilting alone settle down within the first $30 \mathrm{~s}$ after tilting (Toska and Walløe, 2002). Moreover, based on our previous studies we considered that the time required to reach the steady-state responses to changes in PE frequency is $<5$ min (Wieser et al., 2014). Therefore, each combination of angle and PE frequency was measured for $5 \mathrm{~min}$. Measurement at each angle (three PE frequencies at $\alpha=20^{\circ}$ and $\alpha=60^{\circ}, 15 \mathrm{~min}$ for each angle) was preceded by a $5 \mathrm{~min}$ 
measurement period in the supine position. Furthermore, before starting each measurement, an initial 5 min period for a short break and recalibration of the BP monitor (calibration break) was considered. Accordingly, the total measurement time in each experimental session was 40 min per patient (Figure 2). The BP monitor was recalibrated before starting the measurement for each angle.

The measurements were performed on 2 consecutive days. For the experimental session on the first day, the order of presenting the angles and PE frequencies for each patient was randomized. The second-day experiment was a replication of the one of the first day.

Before each experimental session, the patient was positioned on the robot-assisted tilt table by the therapist, and the table was adjusted to patient's height. The legs were placed in the cuffs and adjusted to assure foot loading. The range of motion for hip and knee joints of each leg was measured using the table sensors and provided to the table software to ensure robotic leg movements within these borders. For BP measurement, the arm and finger cuff of the BP monitor were mounted on one arm. An armrest module was used to fix and keep the arm at heart level during measurements.

\subsection{Signal Processing and Statistical Analysis}

The raw $\mathrm{BP}$ signal in the last $2 \mathrm{~min}$ of each experimental condition (i.e., supine, $\mathrm{PE}$ at 0,24 , and 48 steps per min) was analyzed to compute steady-state values of $\mathrm{HR}$, sBP, and dBP (Wieser et al., 2014; Sarabadani Tafreshi et al., 2016). To compute real-time values of sBP (maxima of BP signal) and $\mathrm{dBP}$ (minima of BP signal), the BP signal peaks were identified using a peak detection algorithm in MATLAB ${ }^{\circledR}$ (Mathworks Inc., Natick, MA, United States) and then were visually inspected to ensure correctness and completeness of the detected peaks. Consecutive $\mathrm{dBP}$ peaks were used to compute heart period and accordingly real-time $\mathrm{HR}$ signal. The calculated $\mathrm{HR}, \mathrm{sBP}$, and $\mathrm{dBP}$ biosignals during the last $2 \mathrm{~min}$ of each experimental condition

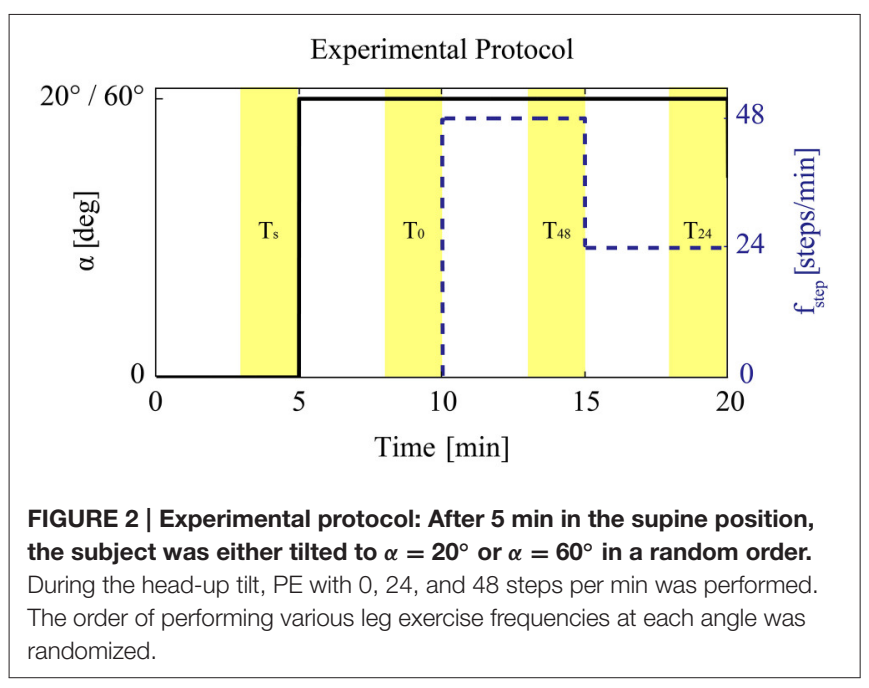

(i.e., $T_{s}, T_{0}, T_{24}, T_{48}$ in Figure 2, corresponding to last 2-min periods of supine, and $\mathrm{PE}$ at 0,24 , and 48 steps per min) were averaged to calculate the steady-state values in each condition (i.e., $\left.\mu_{s}, \mu_{0}, \mu_{24}, \mu_{48}\right)$. In periodic breathing (a phenomenon seen in chronic heart failure patients), the BP oscillations $(0.02-0.4 \mathrm{~Hz}$; Parati et al., 1995) could be overlaid by a very low frequency (VLF) rhythmic pattern (around 0.02 Hz) (Mortara et al., 1997; Francis et al., 2000; Pinna et al., 2000). In such cases, the considered 2 min periods to calculate the steady-state values, do not necessarily include an integer number of VLF cycles. Thus, simple averaging does not cancel the VLF effect, and the calculated steady-state values might not completely represent the changes emerging from PE. Furthermore, our measurements and thus, the considered 2 min periods do not necessarily always start at the same phase of the VLF cycle such that when we, for example, compute the differences between the steady-state values, the VLF effect automatically gets canceled. Therefore, before calculation of steady-state values for these patients, we deployed a fast Fourier transform (FFT) algorithm to find the corresponding frequency of these oscillations related to periodic breathing and then applied moving average windowing with a window size equal to the period of the VLF oscillation. After compensation of the effect of VLF oscillation in the last $2 \mathrm{~min}$ of each condition, we averaged the signals to calculate the steadystate values.

\subsection{Statistical Procedure}

To analyze the repeated measurements and to perform Linear mixed model analyses (Bates et al., 2014) we used R-package (R Core Team, 2015; version 3.2.0). In contrast to repeated measure ANOVA which requires balanced data, linear mixed models can deal with unbalanced data (Verbeke and Molenberghs, 2009). Therefore, this method enabled us to analyze the recorded data from all patients without omitting any data points. The statistical procedure consisted of four steps (Sarabadani Tafreshi et al., 2016):

1. Model assessments: We considered an initial hypothesized model and applied maximum likelihood (ML) algorithm to fit models with a various mixture of fixed and interaction terms (considered in the initial model) to the data. Then we used Akaike information criterion (AIC) and Bayesian information criterion (BIC) scores to compare these models and recognize which main and interaction factors are important to describe the data; those terms which were not significantly improving the fit were removed from the initial model to achieve a final model describing the behavior of each cardiovascular variable with respect to the considered terms.

2. Calculation of $\boldsymbol{p}$-values: Since computation of denominator degrees of freedom for the test statistic in the case of linear mixed models is not straightforward, calculation of $p$-values for these models is a controversy (Bates et al., 2014). However, parametric bootstrap has been suggested as a reliable approach for constructing $p$-values (Bates et al., 2014). Therefore, having found a model with relevant terms, we constructed $p$-values for each relevant term by performing parametric bootstrap (Bates et al., 2014) with 10,000 samples while fitting the models 
using the ML algorithm. For this purpose, we eliminated each relevant term once at a time and using the likelihood ratio test (LRT) and supposing that it has a chi-square distribution, we compared the reduced and full models. Following this, we obtained a chi-square value (observed LRT statistic) from each comparison. Afterward, we randomly generated new data points under the fitted reduced model with one term less (i.e., null hypothesis). We then used the ML algorithm to refit the reduced and full models on this new simulated data [parametric bootstrap (Bates et al., 2014)] and calculated a simulated LRT statistic value by comparing the two models. This scheme was performed 10,000 times resulting in 10,000 simulated LRT statistic values under the null hypothesis. To calculate the corresponding $p$-value for the relevant term under consideration, we then computed the proportion of the simulated LRT statistic values that are bigger or equivalent to the observed LRT statistic value (Halekoh and Højsgaard, 2014). However, this $p$-value calculation approach could not be used when the final model (the result of Step 1) consisted of only an intercept term (the average of data, i.e., the simplest possible model). For such cases, we tested whether the intercept is statistically significantly different from zero. By definition $p$-value for double tailed events can be computed by $2 \times \operatorname{MIN}\left\{\operatorname{Pr}\left(T \leq t_{o b s} ; H_{0}\right), \operatorname{Pr}\left(T \geq t_{o b s} ; H_{0}\right)\right\}$ where $\operatorname{Pr}$ stands for probability, $H_{0}$ for null hypothesis, $T$ is a continuous random variable, and $t_{o b s}$ is an observed value (here, $t_{o b s}=0$ ) (Cox and Hinkley, 1979). Therefore, to calculate the $p$-value examining the significance of the intercept of intercept-only models from zero, we first bootstrapped coefficients for the intercept and computed the fractions of coefficient values higher $\left(p^{+}\right)$and smaller $\left(p^{-}\right)$than zero. We then calculated the $p$-value by multiplying the minimum value of the two fractions by two [i.e., $2 \times \operatorname{MIN}\left(p^{+}, p^{-}\right)$].

3. Final model: Following the calculation of the $p$-values, we used the Restricted Maximum Likelihood (REML) algorithm to refit the model to obtain the model to be reported. To assure the validity of the model we then checked the normality of the residuals by inspecting the corresponding Q-Q plot and performing a Shapiro-Wilk test $(p<0.05$ significant violation). The outcome of this step was the reported model parameter estimates and their standard errors (SE).

4. Confidence intervals: In the last step, we performed another parametric bootstrap with 10,000 samples on the final model (Step 3) to calculate the confidence intervals (CIs) for each model parameter (Bates et al., 2014).

To evaluate the effect of PE, linear mixed model analyses with the dependent variables $\Delta \mathrm{HR}, \Delta \mathrm{sBP}$, and $\Delta \mathrm{dBP}$ (i.e., changes in $\mathrm{HR}$, $\mathrm{sBP}$, and $\mathrm{dBP}$, respectively) were performed in two phases.

\subsection{Primary Analysis}

In the first phase, we focused only on the contribution of PE to the change of cardiovascular variables independent of the head-up tilting effect. To this end, we decoupled the changes introduced by PE from changes introduced by head-up tilting alone. Therefore, we normalized the steady-state values of HR, sBP, and $\mathrm{dBP}$ in response to $24\left(\mu_{24}\right)$ and $48\left(\mu_{48}\right)$ steps per min

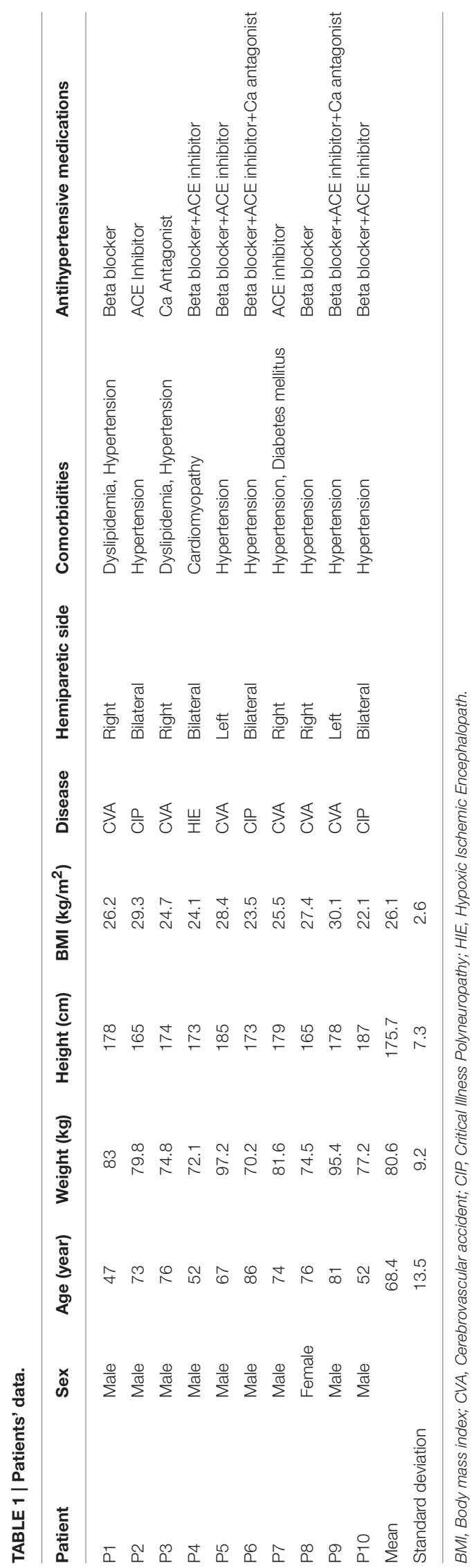


with respect to $0\left(\mu_{0}\right)$ steps per min. This normalization allowed removing the changes introduced by head-up tilting alone from the total changes, and thus, obtaining only changes introduced by PE. Accordingly, the so-called gains of the system, i.e., steadystate changes introduced by only applying the PE at different head-up tilt angles were computed:

$$
\Delta \text { Value }_{24 / 48-0}=\mu_{24 / 48}-\mu_{0}
$$

where $\Delta$ Value corresponds to the relative change in the cardiovascular variable (in $\mathrm{bpm}$ or $\mathrm{mmHg}$ ) by performing $\mathrm{PE}$ at a specific tilt angle. Clearly, the reference value $\left(\mu_{0}\right)$ for each head-up tilt angle experiment was different.

To perform linear mixed model analyses, as fixed effects, the potential effect of tilt angle $(\alpha)$, PE frequency $\left(f_{\text {step }}\right)$, and measurement day were considered into the initial model. The day was treated as a categorical variable referring to measurement day (1 or 2, i.e., first or second day). To have meaningful intercepts in the models that can be interpreted easier, we shifted the angle term with respect to its value at $\alpha=20^{\circ}$ and PE frequency with respect to $f_{\text {step }}=24$ steps per min. Therefore, for the statistical analysis the following initial model was considered:

$$
\begin{array}{r}
\Delta \text { Value }=\beta_{0}+\beta_{1}\left(f_{\text {step }}-24\right)+\beta_{2}\left(\alpha^{\circ}-20^{\circ}\right) \\
+\beta_{3}\left(f_{\text {step }}-24\right) \times\left(\alpha^{\circ}-20^{\circ}\right)+\beta_{4} \times \text { Day }
\end{array}
$$

where $\Delta$ Value corresponds to the relative change in the cardiovascular variable (in bpm or $\mathrm{mmHg}$ ) calculated according to the tilt angle and PE frequency based on equation 1 and $\beta_{i} \mathrm{~s}$ are model coefficients to be estimated. As random effects, we considered two terms; one random intercept for each patient, i.e., (1|patient) to account for potential correlation due to repeated measures of the same patients in various conditions as well as to account for sampling the study population from the general population. Furthermore, one random intercept for each experimental day for each patient (1|patient:day) to account for potential random changes induced in the condition of each experiment by changing the measurement day, such as time of day, meals, etc.

\subsection{Secondary Analysis}

In the second phase, we also considered the head-up tilting effect in the analysis to evaluate whether PE in comparison to headup tilting alone has a significant contribution to the changes in cardiovascular variables and furthermore, whether these changes are in opposite or same directions (compensatory vs. additive). To this end, in contrast to primary analysis where we normalized the steady-state values to 0 steps per $\min \left(\mu_{0}\right)$, we normalized the steady-state values of $\mathrm{HR}, \mathrm{sBP}$, and $\mathrm{dBP}$ in response to $0\left(\mu_{0}\right)$, $24\left(\mu_{24}\right)$, and $48\left(\mu_{48}\right)$ steps per min with respect to the supine position steady-state value $\left(\mu_{s}\right)$. Accordingly, we had:

$$
\Delta \text { Value }_{0 / 24 / 48-s}=\mu_{0 / 24 / 48}-\mu_{s}
$$

where $\Delta$ Value corresponds to the relative change in the cardiovascular variable (in bpm or $\mathrm{mmHg}$ ) with respect to supine position. Clearly, the baseline value $\left(\mu_{s}\right)$ for each head-up tilt angle experiment was different.

The outcome of primary analysis showed that PE frequency did not have a significant effect on the change of steadystate cardiovascular variables, i.e., there was no significant difference between performing $\mathrm{PE}$ at 24 and 48 steps per min (see below, Section 3.2). Therefore, in the secondary analysis, we did not consider the effect of PE frequency but rather considered $\mathrm{PE}$ at both 24 and 48 steps per min, as an occasion of PE. Accordingly, to perform linear mixed model analyses, we considered a categorical variable called PE representing the status of performing PE (equal to 1 for 24 or 48 steps per min, and 0 for 0 steps per min), potential effect of tilt angle $(\alpha)$, and measurement day (treated as categorical variable similar to the primary analysis) as fixed effects into the initial model. To obtain easily interpretable intercepts, the angle term was shifted with respect to its value at $\alpha=20^{\circ}$. Thus, the following initial model was considered for the secondary analysis:

$$
\begin{array}{r}
\Delta \text { Value }=\beta_{0}+\beta_{1} P E+\beta_{2}\left(\alpha^{\circ}-20^{\circ}\right) \\
+\beta_{3} P E \times\left(\alpha^{\circ}-20^{\circ}\right)+\beta_{4} \times \text { Day }
\end{array}
$$

with $\Delta$ Value to be calculated based on equation 3 and $\beta_{i}$ s model coefficients to be estimated. As random effects, similar to the primary analysis we considered two terms; one random intercept for each patient, i.e., (1|patient) and one random intercept for each experimental day for each patient (1|patient:day).

TABLE 2 | Statistical models for $\Delta \mathrm{HR}, \Delta \mathrm{sBP}$, and $\Delta \mathrm{dBP}$ in primary analysis, i.e., consideration of PE effect independent from head-up tilting effect contribution.

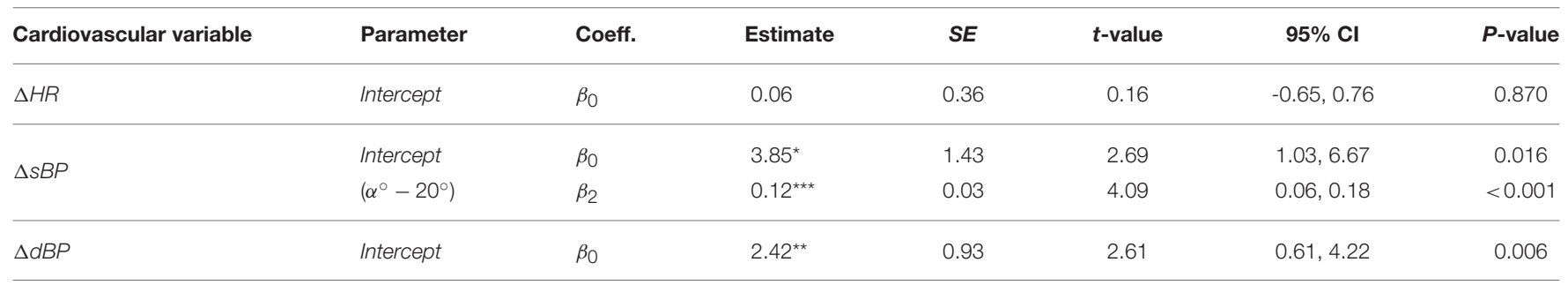

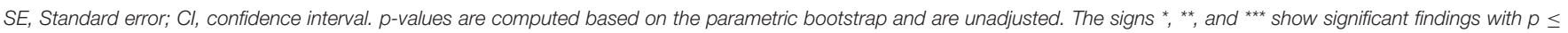
$0.05,0.01$, and 0.001 , respectively. Coeff. parameters represent the coefficients in Equation (2). 


\section{RESULTS}

\subsection{Subjects}

Eleven patients were included in the study. One person with diabetes mellitus was mistakenly included although this was an exclusion criterion. The subjects provided written informed consents before the experiments. From the recruited neurological patients, one subject withdrew his consent after being put on the robot-assisted tilt table. Accordingly, 10 patients participated in the study (nine males and one female; see Table $\mathbf{1}$ for detailed characteristics and demographics). One subject (P6) participated on the first measurement day only. The outcomes of $\alpha=20^{\circ}$ experiment of P6 on day 1 (the only day he had participated) and P7 on day 2 were omitted from analysis due to data collection error. In three subjects (P5, P7, and P10) we compensated observed VLF due to periodic breathing (see above, Section 2.5). We observed no adverse events (e.g., dizziness, presyncope, etc.) related to the orthostatic challenge during the experiments.

\subsection{Primary Analysis}

In the primary analysis, we investigated the effect of PE alone (i.e., independent of the head-up tilting effect). PE had significant contributions to sBP and dBP, but not to HR (see Table 2).

Hypothesis (a): $\mathrm{PE}$ at $\alpha=20^{\circ}$ resulted in significant increase of sBP by about $4 \mathrm{mmHg}$ (see Table 2, $\beta_{0}$, see also Figure 3B). This influence at $\alpha=60^{\circ}$ was pronounced and the amount of increase was significantly higher (about $4.8 \mathrm{mmHg}$ ) as compared to $\alpha=20^{\circ}$, confirming the tilt-angle dependency of the $\mathrm{PE}$ influence on sBP (see Table 2, $\beta_{1}$, see also Figure 3B). For dBP, although $\mathrm{PE}$ in general resulted in significant increase (about $2.5 \mathrm{mmHg}$, see Table 2, $\beta_{0}$, see also Figure 3C), no tilt-angle dependency of the PE influence could be concluded. For HR, no significant contribution was observed (see Table 2, $\beta_{0}$, see also Figure 3A).

Hypothesis (b): Increasing the PE frequency did not result in any significant contribution to the PE effect on any of the cardiovascular variables (neither increase nor reduction of the PE influence). However, when comparing initial models for $\triangle \mathrm{sBP}$ (see above, Step 1 of the statistical procedure in Section 2.6), we found two best models where AIC and BIC scores did not agree. Although, both models provided evidence for tilt-angle dependency of PE influence on $\mathrm{sBP}\left(\beta_{2}\right.$ coefficient in Equation 2), one model also showed a borderline significant trend (parametric bootstrap $p=0.064)$ toward contribution from PE frequency $\left(\beta_{1}\right.$ coefficient in Equation 2) with rather a small effect size $\left(\beta_{1} \simeq\right.$ 0.09 , i.e., $\Delta \mathrm{sBP}=2.2 \mathrm{mmHg}$ ). As the influence of this parameter was rather small, we chose the model with the better (lower) BIC score (i.e., simpler model), only considering the tilt angle influence, to avoid potential overfitting.

Hypothesis (d): Measurement day did not have a significant effect on any variable.

\subsection{Secondary Analysis}

In the secondary analysis we investigated the effect of $\mathrm{PE}$ combined with head-up tilting effect. $\Delta \mathrm{HR}$ at $\alpha=20^{\circ}$ was not influenced significantly by head-up tilting or by PE (see Table 3, $\beta_{0}$, see also Figures $4 \mathbf{A}, \mathbf{B}$ ). We found a significant increase in
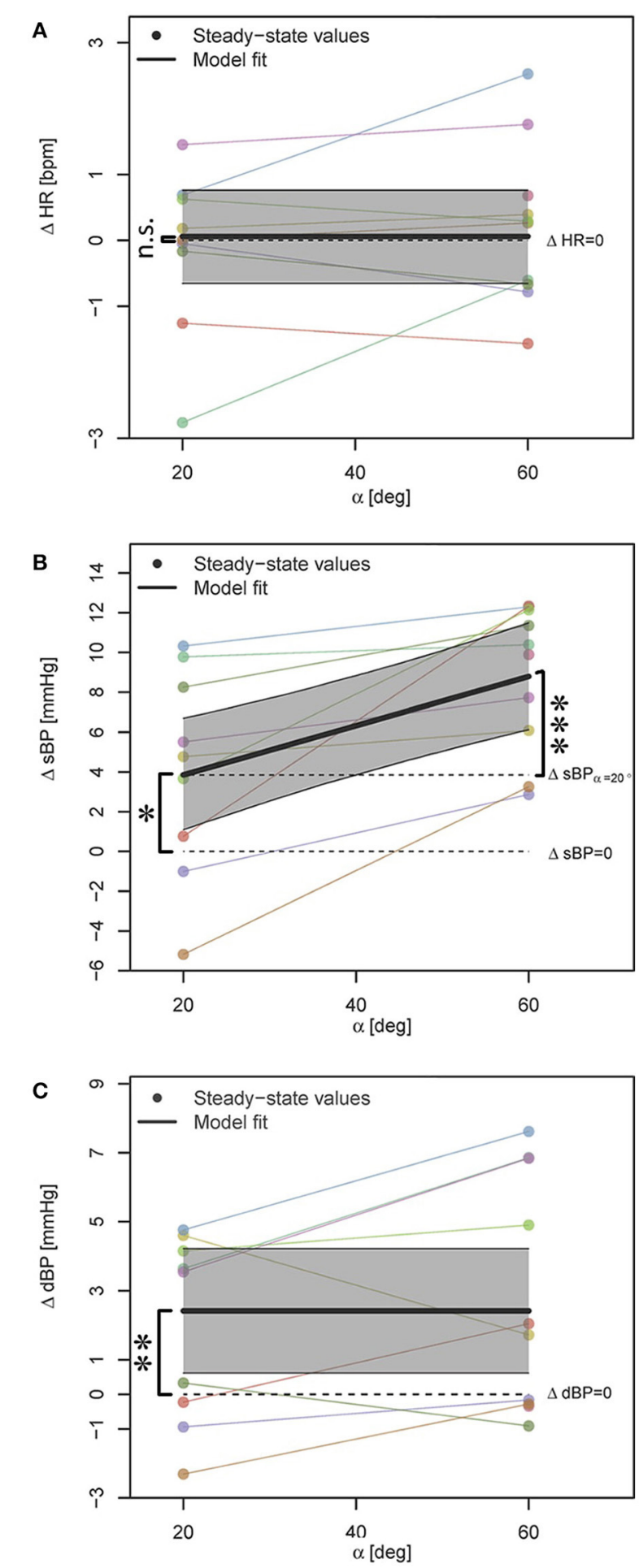

FIGURE 3 | Statistical models for $\Delta \mathrm{HR}, \Delta \mathrm{sBP}$, and $\Delta \mathrm{dBP}$ responses to PE (A-C) when the effect is decoupled from the head-up tilting effect. Data points correspond to the average steady-state response to 24 and 48 steps per min on 2 days. In case of availability of only single case measurement, data point without averaging is shown. The steady-state values for each subject are color-coded and connected with a line. The highlighted areas show 95\% Cl. The signs * ${ }^{* *}$, and ${ }^{* \star *}$ mark significant findings with $p \leq 0.05,0.01$, and 0.001 , respectively. n.s. marks non-significant differences. 
TABLE 3 | Statistical models for $\Delta \mathrm{HR}, \Delta \mathrm{sBP}$, and $\Delta \mathrm{dBP}$ in secondary analysis, i.e., when PE and head-up tilting effects are considered together.

\begin{tabular}{|c|c|c|c|c|c|c|c|}
\hline Cardiovascular variable & Parameter & Coeff. & Estimate & $S E$ & $t$-value & $95 \% \mathrm{Cl}$ & $P$-value \\
\hline \multirow{2}{*}{$\Delta \mathrm{HR}$} & Intercept & $\beta_{0}$ & 1.19 & 0.82 & 1.45 & $-0.41,2.81$ & 0.173 \\
\hline & $\left(\alpha^{\circ}-20^{\circ}\right)$ & $\beta_{2}$ & $0.05^{\star \star \star}$ & 0.01 & 4.50 & $0.03,0.08$ & $<0.001$ \\
\hline \multirow{3}{*}{$\Delta \mathrm{sBP}$} & Intercept & $\beta_{0}$ & -2.58 & 2.24 & -1.15 & $-6.95,1.82$ & 0.239 \\
\hline & $\mathrm{PE}$ & $\beta_{1}$ & $6.45^{\star \star \star}$ & 1.56 & 4.13 & $3.32,9.46$ & $<0.001$ \\
\hline & $\left(\alpha^{\circ}-20^{\circ}\right)$ & $\beta_{2}$ & $0.09^{*}$ & 0.04 & 2.35 & $0.01,0.16$ & 0.023 \\
\hline \multirow{3}{*}{$\Delta \mathrm{dBP}$} & Intercept & $\beta_{0}$ & 1.84 & 1.58 & 1.17 & $-1.27,4.86$ & 0.236 \\
\hline & $\mathrm{PE}$ & $\beta_{1}$ & $2.55^{\star}$ & 1.19 & 2.14 & $0.25,4.86$ & 0.036 \\
\hline & $\left(\alpha^{\circ}-20^{\circ}\right)$ & $\beta_{2}$ & $0.22^{\star \star \star}$ & 0.03 & 7.64 & $0.16,0.27$ & $<0.001$ \\
\hline
\end{tabular}

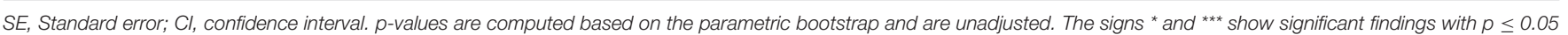
and 0.001 , respectively. Coeff. parameters represent the coefficients in Equation (4).

HR when tilted to $\alpha=60^{\circ}$ in comparison to $\alpha=20^{\circ}$ (about 2 bpm, see Table 3, $\beta_{2}$, see also Figures 4A,B) but PE did not have a significant contribution to the changes (see Table 3 , see Figures $4 \mathbf{A}, \mathbf{B}$ ). This was expected as in the primary analysis no systematic contribution of PE on $\triangle \mathrm{HR}$ was observed (see Figure 3A).

Hypothesis (c): Head-up tilting alone (without PE) to $\alpha=20^{\circ}$ had no statistically significant effect on $\mathrm{sBP}$ (see Table $3, \beta_{0}$, see also Figures 4C,D). We observed a trend toward sBP drop at $\alpha=20^{\circ}$ (without $\mathrm{PE}$ ) as compared to the supine position. No drop at $\alpha=60^{\circ}$ (without PE) was observed (see Figures 4C,D). $\triangle \mathrm{sBP}$ increased significantly with $\mathrm{PE}$ (about $6.45 \mathrm{mmHg}$, see Table 3, $\beta_{1}$, see also Figures 4C,D) and with change in tilt angle from $\alpha=20^{\circ}$ to $\alpha=60^{\circ}$ (about $3.6 \mathrm{mmHg}$, see Table 3, $\beta_{2}$, see also Figures 4 C,D). These results show that PE had a very slight compensatory (only at $\alpha=20^{\circ}$ ) but more additive effect on increasing sBP (see Figures 4C,D) in our study population.

The effect of head-up tilting alone (without PE) to $\alpha=20^{\circ}$ on $\mathrm{dBP}$ was not statistically significant although a positive trend could be observed (see Table 3, $\beta_{0}$, see also Figures $4 \mathrm{E}, \mathrm{F}$ ). PE and change in head-up tilt angle from $\alpha=20^{\circ}$ to $\alpha=60^{\circ}$ both resulted in statistically significant increase of dBP (see Table 3, $\beta_{1}$ and $\beta_{2}$, respectively, see also Figures $4 \mathbf{E}, \mathbf{F}$ ) with about 2.5 and $8.8 \mathrm{mmHg}$ increase, respectively. This clearly shows the additive effect of PE on the head-up tilting effect on increasing $\mathrm{dBP}$.

Hypothesis (d): Measurement day did not have a significant effect in any case.

\section{DISCUSSION}

We observed that PE alone increased $\mathrm{sBP}$ and $\mathrm{dBP}$ in a statistically significant manner but had no significant effect on HR during head-up tilt in neurological patients. Only for sBP, the effect was dependent on the tilt angle, i.e., performing $\mathrm{PE}$ at higher angles resulted in higher increase in sBP.

In our previous study in a healthy population (Sarabadani Tafreshi et al., 2016), we had observed similar but to some extent different results: PE during head-up tilt had resulted in significant changes in $\mathrm{HR}, \mathrm{sBP}$, and $\mathrm{dBP}$. However, changes in both, sBP and $\mathrm{dBP}$ were dependent on the tilt angle. Furthermore, these changes were not always in the same direction (i.e., increase or decrease of the cardiovascular variables) (Sarabadani Tafreshi et al., 2016). In the current study, the contribution of PE on $\mathrm{sBP}$ and $\mathrm{dBP}$ (independent from the tilt angle) was always positive and in the same direction, resulting in significant increases of sBP and $\mathrm{dBP}$. This observation might explain the protective effect of $\mathrm{PE}$ on orthostatic hypotension: significantly fewer people (both, healthy subjects and patients) experience orthostatic hypotension on a robot-assisted tilt table where PE is included (as compared to a standard tilt table) at a tilt angle between $\alpha=60^{\circ}-75^{\circ}$ (Czell et al., 2004; Luther et al., 2008; Kuznetsov et al., 2013).

Although the majority of patients took cardioactive medication (e.g., beta blockers in 7 out of 10 subjects, see Table 1), the amount of change in BP was relatively higher to that of healthy subjects. However, in contrast to healthy subjects, in the patients, we did not observe any significant changes in $\mathrm{HR}$ in response to PE. We only observed a slight increase of HR in response to head-up tilting (about $3.2 \mathrm{bpm}$ see Table 3, $\Delta$ HR model). In our former study on healthy subjects (Sarabadani Tafreshi et al., 2016), we had observed either no significant change (at $\alpha=20^{\circ}$ ) or significant reduction of $\mathrm{HR}$ at tilt angles above $\alpha=20^{\circ}$ (Sarabadani Tafreshi et al., 2016) in response to $\mathrm{PE}$ alone, and a significant increase in response to head-up tilting alone (average $16 \mathrm{bpm}$ at $\alpha=60^{\circ}$ ). It is known that the baroreflex buffers fluctuations of BP that occur due to changes in position [e.g., head-up tilting (Schwartz and Stewart, 2012)], exercise (Raven et al., 2006) or other conditions. It can be depressed in older people (Jones et al., 2003) or patients with neurological disorder (Benarroch, 2008), leading to an increase of peripheral resistance and higher HR (Benarroch, 2008). Thus, we expected in our study population a baroreflex depression and consequently more pronounced changes in $\mathrm{BP}$ and $\mathrm{HR}$. However, we only observed that in case of BP.

Furthermore, we looked at the effect of the change in $\mathrm{PE}$ frequency on the cardiovascular system. Only for sBP, we observed a trend toward higher values, but the effect was not clinically relevant (i.e., $\mathrm{HR}>2.5 \mathrm{bpm}$ and $\mathrm{BP}>5 \mathrm{mmHg}$ Sarabadani Tafreshi et al., 2017b) even when we doubled the 

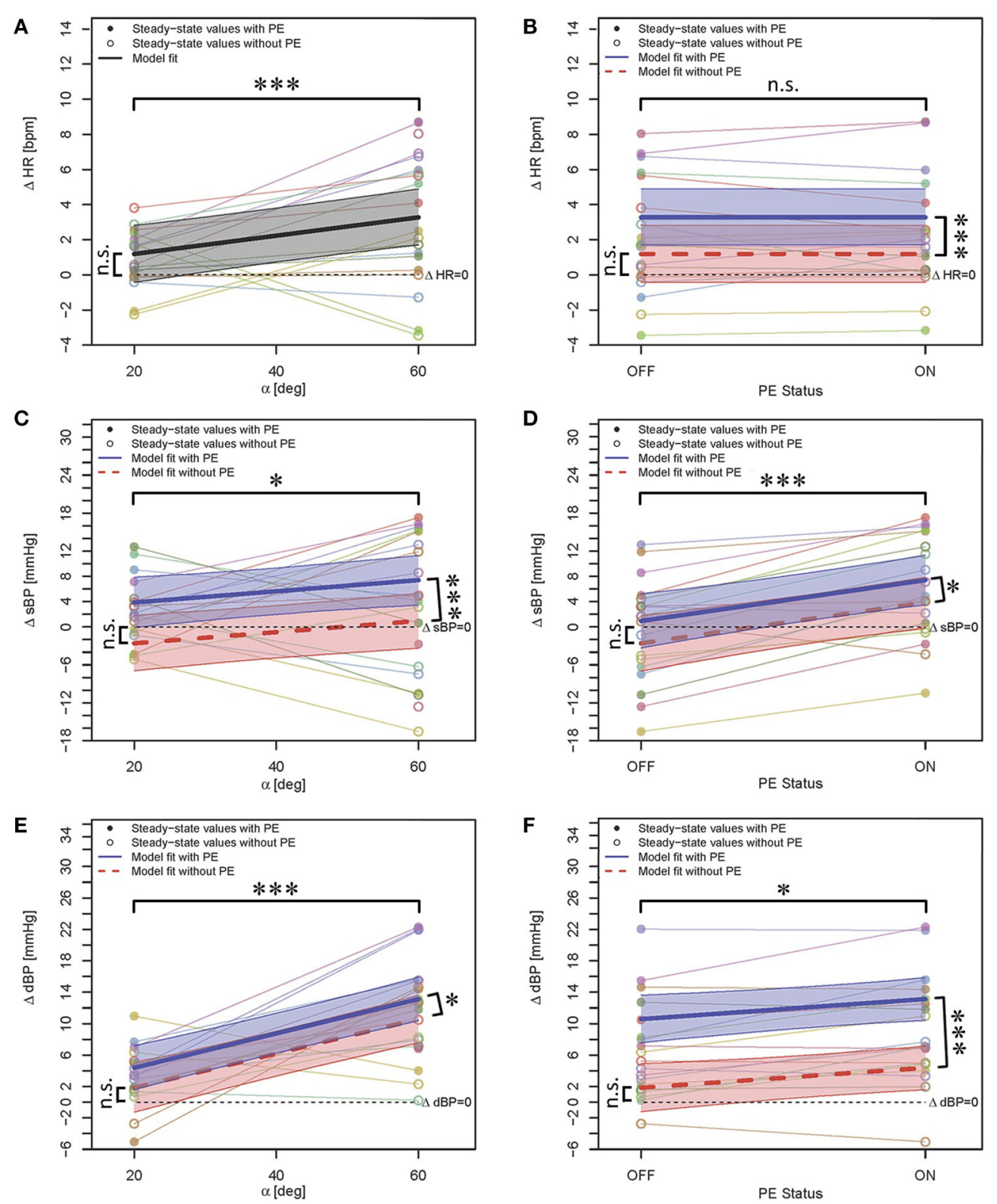

FIGURE 4 | Statistical models for $\Delta \mathbf{H R}(\mathbf{A}, \mathbf{B}), \Delta \mathrm{SBP}(\mathbf{C}, \mathbf{D})$, and $\Delta \mathrm{dBP}(\mathbf{E}, \mathbf{F})$ responses to $P E$ when the effect is considered together with the effect of head-up tilting. In this figure, both columns show the same models with variation with respect to tilt angle (left column) and PE status (right column), respectively. As the PE effect for $\triangle \mathrm{HR}$ was not significant, Figure 4A, only shows one model for both conditions of with and without PE. Data points correspond to the average of steady-state response on two days. In case of availability of only single case measurement, the data point without averaging is shown. The steady-state values for each subject in each case (PE status or tilt angle) are color-coded and connected with lines. The highlighted areas show $95 \% \mathrm{Cl}$. The signs * and ${ }^{\star \star *}$ mark significant findings with $p \leq 0.05$ and 0.001 , respectively. n.s. marks non-significant differences.

PE frequency from 24 to 48 steps per min. This result is in contrast to the current belief in the clinical application of robotassisted tilt tables that higher PE frequency would result in higher effects. We conclude that the relationship of cardiovascular variables and $\mathrm{PE}$ is not proportional, i.e., PE frequency does not scale to cardiovascular variables. Rather, it reaches a plateau where a higher increase of PE frequency would not result in any systematic cardiovascular changes. Still, PE (independent of its frequency), increases $\mathrm{sBP}$ and $\mathrm{dBP}$ and does so especially at high tilt angles. This effect might have the potential to compensate the drop in $\mathrm{BP}$ due to orthostatic challenge and, as a consequence, prevent syncope during head-up tilt. This is a consistent conclusion also supported by previous studies (Chi et al., 2008; Yoshida et al., 2013).

When combining PE with head-up tilting, PE had an additive effect on increasing both sBP and dBP. However, as the mean 
BP drop in response to head-up tilting in our study was not significant, we can neither conclude nor reject that $\mathrm{PE}$ could have a systematic compensatory effect to prevent $\mathrm{sBP}$ or $\mathrm{dBP}$ drop and potentially syncope.

The day effect was not significant in any of the analyses, indicating that the observed effects of PE on the cardiovascular variables are consistent from day to day. However, the result mirrors the average, and it cannot be concluded that PE always changes these variables in the same direction or same amount in a single subject.

We developed independent statistical models for relative changes of $\mathrm{HR}, \mathrm{sBP}$, and $\mathrm{dBP}$, although these signals were recorded during the same experiments. We did not correct the reported $p$-values for multiplicity (Sare et al., 2009) because the cardiovascular variables are coupled and various mechanisms such as the baroreflex circuit (Jones et al., 2003; Mukkamala et al., 2003; Raven et al., 2006; Benarroch, 2008; Schwartz and Stewart, 2012) govern the relationship between them. However, the degree of this dependency during head-up tilt or PE is not clear to us. In particular, baroreflex resetting [e.g., during exercise (Raven et al., 2006) or orthostatic challenge (Schwartz and Stewart, 2012)] makes investigation of such dependencies more challenging. Therefore, although we acknowledge this limitation of our study, we avoided any systematic correction for the multiplicity of our reported $p$-values.

\section{CONCLUSION AND OUTLOOK}

We provide evidence that $\mathrm{PE}$ can increase $\mathrm{sBP}$ and $\mathrm{dBP}$ of neurological patients during head-up tilt. However, in our population with mild BP changes, we could not conclude a preventive effect of $\mathrm{PE}$ on orthostatic hypotension and syncope. A study with a more homogenous patient population is necessary to underpin the preventive effect of PE on orthostatic

\section{REFERENCES}

Bates, D., Mächler, M., Bolker, B., and Walker, S. (2014). Fitting linear mixedeffects models using lme4. arXiv:1406.5823.

Benarroch, E. E. (2008). The arterial baroreflex functional organization and involvement in neurologic disease. Neurology 71, 1733-1738. doi: 10.1212/01.wnl.0000335246.93495.92

Bourdin, G., Barbier, J., Burlem, J.-F., Durante, G., Passant, S., Vincent, B., et al. (2010). The feasibility of early physical activity in intensive care unit patients: a prospective observational one-center study. Respir. Care 55, 400-407.

Brower, R. G. (2009). Consequences of bed rest. Crit. Care Med. 37, S422-S428. doi: 10.1097/CCM.0b013e3181b6e30a

Burtin, C., Clerckx, B., Robbeets, C., Ferdinande, P., Langer, D., Troosters, T., et al. (2009). Early exercise in critically ill patients enhances short-term functional recovery. Crit. Care Med. 37, 2499-2505. doi: 10.1097/CCM.0b013e3181a 38937

Chi, L., Masani, K., Miyatani, M., Thrasher, T. A., Johnston, K. W., Mardimae, A., et al. (2008). Cardiovascular response to functional electrical stimulation and dynamic tilt table therapy to improve orthostatic tolerance. J. Electromyogr. Kinesiol. 18, 900-907. doi: 10.1016/j.jelekin.2008.08.007

Colombo, G., Schreier, R., Mayr, A., Plewa, H., and Rupp, R. (2005). "Novel tilt table with integrated robotic stepping mechanism: design principles and clinical application," in 9th International Conference on Rehabilitation Robotics, 2005, ICORR 2005 (Chicago, IL: IEEE), 227-230. hypotension and syncope in neurological patients. Furthermore, we observed that similar to healthy subjects, the effect of PE on sBP is dependent on the verticalization angle.

To our knowledge, this is the first study which systematically investigated the effect of PE frequency on the cardiovascular system's response. We found that modulation of the PE frequency does not play an important role in PE influence. Hence, PE frequency cannot be used as a reliable variable to modulate PE influence. Thus, investigation of other modalities such as foot loading to modulate and enhance the PE effect is a potential future work.

\section{AUTHOR CONTRIBUTIONS}

AS, RR, and VK conceived the study. AS designed and coordinated the study, and collected and analyzed the data. AS, $\mathrm{RR}$, and VK interpreted the data. AS wrote the first draft of the manuscript. AS, RR, and VK revised the manuscript.

\section{FUNDING}

The financial support of this research was provided by the Commission for Technology and Innovation CTI, Switzerland, and the European Community's Seventh Framework Programme FP7/2007-2013 under grant agreement no. 312815-STAMAS.

\section{ACKNOWLEDGMENTS}

Special thanks go to Maya Ida Kamber and Ingmar Anner for their clinical support during the study as well as all the patients who kindly participated. We acknowledge fruitful discussions with Amir Esmaeil Sarabadani Tafreshi (the Institute of Information Systems, ETH Zürich) and Francesco Crivelli (the Sensory-Motors Systems Lab, ETH Zürich).
Cox, D. R., and Hinkley, D. V. (1979). Theoretical Statistics. CRC Press.

Craven, C. T. D., Gollee, H., Coupaud, S., and Allan, D. B. (2013). Investigation of robotic-assisted tilt-table therapy for early-stage spinal cord injury rehabilitation. J. Rehab. Res. Dev. 50, 367-378. doi: 10.1682/JRRD.2012. 02.0027

Czell, D., Schreier, R., Rupp, R., Eberhard, S., Colombo, G., and Dietz, V. (2004). Influence of passive leg movements on blood circulation on the tilt table in healthy adults. J. Neuroeng. Rehab. 1:1. doi: 10.1186/1743-0003-1-4

Dittmer, D., and Teasell, R. (1993). Complications of immobilization and bed rest. part 1: musculoskeletal and cardiovascular complications. Can. Fam. Physician 39:1428.

Feldstein, C., and Weder, A. B. (2012). Orthostatic hypotension: a common, serious and underrecognized problem in hospitalized patients. J. Am. Soc. Hypertens. 6, 27-39. doi: 10.1016/j.jash.2011.08.008

Francis, D. P., Davies, L. C., Willson, K., Ponikowski, P., Coats, A. J., and Piepoli, M. (2000). Very-low-frequency oscillations in heart rate and blood pressure in periodic breathing: role of the cardiovascular limb of the hypoxic chemoreflex. Clin. Sci. 99, 125-132. doi: 10.1042/cs0990125

Frazzitta, G., Valsecchi, R., Zivi, I., Sebastianelli, L., Bonini, S., Zarucchi, A., et al. (2015). Safety and feasibility of a very early verticalization in patients with severe traumatic brain injury. J. Head Trauma Rehab. 30, 290-292. doi: 10.1097/HTR.0000000000000135

Freeman, R., Wieling, W., Axelrod, F. B., Benditt, D. G., Benarroch, E., Biaggioni, I., et al. (2011). Consensus statement on the definition of orthostatic hypotension, 
neurally mediated syncope and the postural tachycardia syndrome. Clin. Auton. Res. 21, 69-72. doi: 10.1007/s10286-011-0119-5

Giggins, O. M., Persson, U. M., and Caulfield, B. (2013). Biofeedback in rehabilitation. J. Neuroeng. Rehab. 10:1. doi: 10.1186/1743-0003-10-60

Halekoh, U., and Højsgaard, S. (2014). A kenward-roger approximation and parametric bootstrap methods for tests in linear mixed models-the $\mathrm{r}$ package pbkrtest. J. Stat. Softw. 59, 1-30. doi: 10.18637/jss.v059.i09

Illman, A., Stiller, K., and Williams, M. (2000). The prevalence of orthostatic hypotension during physiotherapy treatment in patients with an acute spinal cord injury. Spinal Cord 38, 741-747. doi: 10.1038/sj.sc.3101089

Jones, P. P., Christou, D. D., Jordan, J., and Seals, D. R. (2003). Baroreflex buffering is reduced with age in healthy men. Circulation 107, 1770-1774. doi: 10.1161/01.CIR.0000057811.86187.88

Kuznetsov, A. N., Rybalko, N. V., Daminov, V. D., and Luft, A. R. (2013). Early poststroke rehabilitation using a robotic tilt-table stepper and functional electrical stimulation. Stroke Res. Treat. 2013:946056. doi: 10.1155/2013/946056

Laubacher, M., Perret, C., and Hunt, K. J. (2015). Work-rate-guided exercise testing in patients with incomplete spinal cord injury using a robotics-assisted tilt-table. Disabil. Rehab. Assist. Technol. 10, 433-438. doi: 10.3109/17483107.2014.908246

Luther, M. S., Krewer, C., Müller, F., and Koenig, E. (2008). Comparison of orthostatic reactions of patients still unconscious within the first three months of brain injury on a tilt table with and without integrated stepping. a prospective, randomized crossover pilot trial. Clin. Rehab. 22, 1034-1041. doi: 10.1177/0269215508092821

Morris, P. E. (2007). Moving our critically ill patients: mobility barriers and benefits. Crit. Care Clin. 23, 1-20. doi: 10.1016/j.ccc.2006.11.003

Mortara, A., Sleight, P., Pinna, G. D., Maestri, R., Prpa, A., La Rovere, M. T., et al. (1997). Abnormal awake respiratory patterns are common in chronic heart failure and may prevent evaluation of autonomic tone by measures of heart rate variability. Circulation 96, 246-252. doi: 10.1161/01.CIR.96.1.246

Mukkamala, R., Toska, K., and Cohen, R. J. (2003). Noninvasive identification of the total peripheral resistance baroreflex. Am. J. Physiol. Heart Circul. Physiol. 284, H947-H959. doi: 10.1152/ajpheart.00532.2002

Pagani, M., Lombardi, F., Guzzetti, S., Rimoldi, O., Furlan, R., Pizzinelli, P., et al. (1986). Power spectral analysis of heart rate and arterial pressure variabilities as a marker of sympatho-vagal interaction in man and conscious dog. Circul. Res. 59, 178-193.

Parati, G., Saul, J. P., Di Rienzo, M., and Mancia, G. (1995). Spectral analysis of blood pressure and heart rate variability in evaluating cardiovascular regulation a critical appraisal. Hypertension 25, 1276-1286. doi: 10.1161/01.HYP.25.6.1276

Pinna, G., Maestri, R., Mortara, A., and La Rovere, M. (2000). Cardiorespiratory interactions during periodic breathing in awake chronic heart failure patients. Am. J. Physiol. Heart Circul. Physiol. 278, H932-H941.

R Core Team (2015). R: A Language and Environment for Statistical Computing. Vienna: R Foundation for Statistical Computing.

Raven, P. B., Fadel, P. J., and Ogoh, S. (2006). Arterial baroreflex resetting during exercise: a current perspective. Exp. Physiol. 91, 37-49. doi: 10.1113/expphysiol.2005.032250

Ravensbergen, H. J. R., de Groot, S., Post, M. W., Slootman, H. J., van der Woude, L. H., and Claydon, V. E. (2013). Cardiovascular function after spinal cord injury prevalence and progression of dysfunction during inpatient rehabilitation and 5 years following discharge. Neurorehab. Neural Repair 28, 219-229. doi: 10.1177/1545968313504542

Saengsuwan, J., Huber, C., Schreiber, J., Schuster-Amft, C., Nef, T., and Hunt, K. J. (2015a). Feasibility of cardiopulmonary exercise testing and training using a robotics-assisted tilt table in dependent-ambulatory stroke patients. J. Neuroeng. Rehab. 12:1. doi: 10.1186/s12984-015-0078-5

Saengsuwan, J., Nef, T., Laubacher, M., and Hunt, K. J. (2015b). Comparison of peak cardiopulmonary performance parameters on a roboticsassisted tilt table, a cycle and a treadmill. PLoS ONE 10:e122767. doi: 10.1371/journal.pone.0122767
Sarabadani Tafreshi, A., Klamroth-Marganska, V., Nussbaumer, S., and Riener, R. (2015). Real-time closed-loop control of human heart rate and blood pressure. IEEE Trans. Biomed. Eng. 62, 1434-1442. doi: 10.1109/TBME.2015.23 91234

Sarabadani Tafreshi, A., Okle, J., Klamroth-Marganska, V., and Riener, R. (2017a). Modeling the effect of tilting, passive leg exercise, and functional electrical stimulation on the human cardiovascular system. Med. Biol. Eng. Comput. doi: 10.1007/s11517-017-1628-8. [Epub ahead of print].

Sarabadani Tafreshi, A., Riener, R., and Klamroth-Marganska, V. (2016). Distinctive steady-state heart rate and blood pressure responses to passive robotic leg exercise and functional electrical stimulation during head-up tilt. Front. Physiol. 7:612. doi: 10.3389/fphys.2016.00612

Sarabadani Tafreshi, A., Riener, R., and Klamroth-Marganska, V. (2017b). Quantitative analysis of externally-induced patterns and natural oscillations in the human cardiovascular response: Implications for development of a biofeedback system. Biomed. Signal Process. Control 36, 76-83. doi: 10.1016/j.bspc.2017.03.021

Sare, G. M., Ali, M., Shuaib, A., Bath, P. M., and VISTA Collaboration (2009). Relationship between hyperacute blood pressure and outcome after ischemic stroke data from the vista collaboration. Stroke 40, 2098-2103. doi: 10.1161/STROKEAHA.108.539155

Schwartz, C. E., and Stewart, J. M. (2012). The arterial baroreflex resets with orthostasis. Front. Physiol. 3:461. doi: 10.3389/fphys.2012. 00461

Taveggia, G., Ragusa, I., Trani, V., Cuva, D., Angeretti, C., Fontanella, M., et al. (2015). Robotic tilt table reduces the occurrence of orthostatic hypotension over time in vegetative states. Int. J. Rehab. Res. 38, 162-166. doi: 10.1097/MRR.0000000000000104

Teasell, R., and Dittmer, D. (1993). Complications of immobilization and bed rest. part 2: other complications. Can. Fam. Physician 39:1440.

Toska, K., and Walløe, L. (2002). Dynamic time course of hemodynamic responses after passive head-up tilt and tilt back to supine position. J. Appl. Physiol. 92, 1671-1676. doi: 10.1152/japplphysiol.00465.2000

Verbeke, G., and Molenberghs, G. (2009). Linear Mixed Models for Longitudinal Data. New York, NY: Springer Science \& Business Media.

West, C. R., Crawford, M. A., Laher, I., Ramer, M. S., and Krassioukov, A. V. (2015). Passive hind-limb cycling reduces the severity of autonomic dysreflexia after experimental spinal cord injury. Neurorehab. Neural Repair 30, 317-327. doi: $10.1177 / 1545968315593807$

Wieser, M., Gisler, S., Sarabadani, A., Ruest, R. M., Buetler, L., Vallery, H., et al. (2014). Cardiovascular control and stabilization via inclination and mobilization during bed rest. Med. Biol. Eng. Comput. 52, 53-64. doi: 10.1007/s11517-013-1119-5

Wieser, M. J. (2011). A Multi-Modal Approach to Improve the Rehabilitation Therapy of Bed Rest Patients. Ph.D. thesis, Diss., Eidgenössische Technische Hochschule ETH, Nr. 20032, Zürich.

Yoshida, T., Masani, K., Sayenko, D. G., Miyatani, K., Fisher, J. A., and Popovic, M. R. (2013). Cardiovascular response of individuals with spinal cord injury to dynamic functional electrical stimulation under orthostatic stress. IEEE Trans. Neural Syst. Rehab. Eng. 21, 37-46. doi: 10.1109/TNSRE.2012.22 11894

Conflict of Interest Statement: The authors declare that the research was conducted in the absence of any commercial or financial relationships that could be construed as a potential conflict of interest.

Copyright (c) 2017 Sarabadani Tafreshi, Riener and Klamroth-Marganska. This is an open-access article distributed under the terms of the Creative Commons Attribution License (CC BY). The use, distribution or reproduction in other forums is permitted, provided the original author(s) or licensor are credited and that the original publication in this journal is cited, in accordance with accepted academic practice. No use, distribution or reproduction is permitted which does not comply with these terms. 